\title{
ANÁLISE DE AGRUPAMENTO APLICADA AO RECONHECIMENTO DE DIFERENTES TIPOS DE ITABIRITOS SILICOSOS FRIÁVEIS DA MINA DE BRUCUTU
}

\author{
Gizele Maria Campos Gonçalves ' \\ Geriane Macedo Rocha' \\ Paula Bernardes Barreto ' \\ Tiago Martins Pereira ' \\ Rosa Malena Fernandes Lima'
}

\section{Resumo}

A análise de agrupamento é uma técnica de estatística multivariada que permite separar as unidades amostrais em grupos homogêneos internamente e heterogêneos externamente. Neste estudo essa técnica foi aplicada, através do software R, com o objetivo de estratificar em subgrupos os minérios classificados como itabiritos silicosos friáveis da Mina de Brucutu, Quadrilátero Ferrífero-MG. A matriz de dados era composta por 3.519 amostras e 10 variáveis granulométricas e químicas. A análise hierárquica de agrupamento mostrou que as amostras podem ser subdivididas em quatro grupos, e então, as mesmas foram agrupadas pelo método não hierárquico $K$-means. Através da análise das características de cada grupo formado, os mesmos foram classificados e relacionados com a geologia da jazida. A Tipologia I (itabirito silicoso friável rico e fino), é localizada no topo do depósito e estratigraficamente acima das rochas intrusivas máficas. A Tipologia 2 (itabirito silicoso friável pobre e grosseiro) é marcado pela direção NW-SE, evento Brasiliano. A Tipologia 3 (itabirito silicoso friável pobre e fino) é dispersa ao longo do depósito, dificultando sua correlação com alguma fase geológica. A Tipologia 4 (itabirito semi-compacto pobre) concentra-se na região SW do depósito.

Palavras-chave: Análise de agrupamento; Minério de ferro; K-means.

\section{CLUSTER ANALYSIS APPLIED TO RECOGNITION OF DIFFERENT TYPES OF FRIABLE SILICEOUS ITABIRITES FROM BRUCUTU MINE}

\begin{abstract}
Cluster analysis is a multivariate statistical technique that allows to separate the sample units into internally homogeneous and externally heterogeneous groups. In this study the cluster analysis was applied through $\mathrm{R}$ software, with the aim of stratifying into subgroups the ores classified as friable siliceous itabirite from the Brucutu Mine, Quadrilátero Ferrífero, state of Minas Gerais, Brazil. The data matrix consists of 3519 samples and 10 variables related to granulometric and chemical data. The hierarchical clustering analysis showed that the samples could be subdivided into four groups, and then they were grouped by the non-hierarchical K-means method. Through the analysis of the features of each group formed they were classified and related to the geology of the deposit. Typology I (friable, Fe-rich and fine siliceous itabirite) is located at the top of the deposit and stratigraphically above the intrusive mafic rocks. Typology 2 (- friable, low grade and coarse siliceous itabirite) is marked by NW-SE direction, Brasiliano event. Typology 3 (friable, low grade and fine siliceous itabirite) is dispersed throughout the deposit, making it difficult to correlate with some geological phase. Typology 4 (low grade semi-compact itabirite) is demarcated by concentration in the SW region of the reservoir.
\end{abstract}

Keywords: Iron ore; Cluster analysis; K-means.

\section{INTRODUÇÃO}

A identificação de diferentes tipologias de minérios, com base em suas características intrínsecas, é fundamental tanto para quantificação de reservas quanto para otimização de circuitos industriais de beneficiamento, uma vez que pequenas alterações nas suas propriedades físicas e químicas podem influenciar nos resultados de operações unitárias

'Programa de Pós-graduação em Engenharia Mineral, Departamento de Engenharia de Minas, Universidade Federal de Ouro Preto, Ouro Preto, MG, Brasil. E-mail: gizele.campos@vale.com 
(fragmentação/classificação e concentração) às quais os mesmos serão submetidos [I]. Sendo assim, métodos que identifiquem populações tipológicas com características similares, como é o caso do método estatístico de análise multivariada por agrupamento, ou cluster analysis, podem ser de grande importância para o entendimento e zoneamento da mina, visando o planejamento de lavra e, consequentemente o beneficiamento de um dado minério.

Em empreendimentos minerários, as formações ferríferas (itabiritos), são classificadas com base nas suas composições mineralógicas e propriedades físicas. Dentre essas classificações algumas principais são: itabiritos goethíticos (friável ou compacto), itabirito silicoso (friável ou compacto), itabirito silicoso semi-compacto, itabirito silicoso compacto e itabirito manganesífero [2]. De acordo com a composição química, especialmente teor de $\mathrm{Fe}$, os itabiritos podem ser divididos em pobres $\mathrm{Fe}<40 \%$ e ricos $\mathrm{Fe}>55 \%$ [3]. Dependendo do grau de alteração, os minérios de ferro podem ser considerados mais ou menos hidratados, o que é indicado pelo PPC (perda por calcinação) $>3 \%$ e ou contaminados por $\mathrm{Al}_{2} \mathrm{O}_{3}, \mathrm{P}$ e/ou Mn [4,5]. Sousa [6] explica que estas diferenças estão relacionadas com as condições geológicas a que os mesmos foram submetidos, tal como intempéries climáticas, que propiciaram um enriquecimento supergênico, ocasionado por condições de chuvas intensas e elevadas temperaturas. A maioria dos depósitos classificados como de enriquecimento supergênico ocorre em topografias elevadas que, geomorfologicamente, são classificadas como colinas, montanhas ou planaltos, cujas alturas variam de $600 \mathrm{~m}$ a $1000 \mathrm{~m}$. Neste tipo de depósito, o enriquecimento supergênico pode chegar até profundidades de $200 \mathrm{~m}$, como - caso dos depósitos de Carajás, Quadrilátero Ferrífero, Província de Hamersley e Simandu [7]. Para um enriquecimento supergênico eficaz as camadas de formações ferríferas bandadas (BIF's) agem como um aquífero confinado, ou parcialmente confinado, por unidades impermeáveis como xistos, rochas máficas ou zonas milonitizadas de grãos finos. Endo et al. [8] descrevem que as rochas máficas presentes no depósito de Brucutu ocorrem de forma concordante ao bandamento composicional dos itabiritos, sendo tratada como uma barreira hidráulica. Neste sentido, o intemperismo provocado pelas águas meteóricas permitiu a infiltração nestas BIF's até a zona impermeável, provocando a lixiviação da sílica e carbonatos levando à concentração de ferro nas condições oxidantes, tanto residual como a hematita e magnetita, quanto na formação de novos minerais (hematita, maghemita e goethita). Localmente ainda pode ocorrer a formação de óxidos e silicatos de alumina como a gibbsita e caulinita, e oxi-hidróxidos de manganês. Este intemperismo permitiu a formação de um minério friável, por vezes pulverulento, mas que ainda localmente podem envelopar minérios maciços e compactos reliquiares [7].

Este trabalho teve como objetivo realizar a estratificação em subgrupos de itabiritos silicosos friáveis pela aplicação de ferramenta de análise multivariada (análise de agrupamento, utilizando o algoritmo $K$-means) baseando-se em dados de análises granulométricas e químicas de amostras de minério de ferro da Mina de Brucutu, localizada no extremo leste do Quadrilátero Ferrífero.

\section{MATERIAIS E MÉTODOS}

A matriz de dados é representada por 10 variáveis e 3.519 amostras classificadas como itabirito silicoso friável, coletadas através de sondagem rotativa diamantada ( $32 \%$ das amostras) e amostragem de canaletas nas frentes de lavra (68\% das amostras). A disposição espacial dessas amostras está em um horizonte de 10 metros acima ou abaixo da superfície topográfica da mina referente a janeiro de 2018. As 10 variáveis correspondem às principais características granulométricas e químicas do minério: $+8,0 \mathrm{~mm},-8,0+1,0 \mathrm{~mm}$, $-\mathrm{I}, 0+0,15 \mathrm{~mm},-0,15 \mathrm{~mm}, \mathrm{Fe}, \mathrm{SiO}_{2}, \mathrm{Al}_{2} \mathrm{O}_{3}, \mathrm{P}, \mathrm{Mn}$ e PPC.

A distribuição granulométrica das amostras foi determinada por peneiramento à seco por 20 minutos (Laboratório Físico de Brucutu), usando as seguintes peneiras: $8,0 \mathrm{~mm}, 4,0 \mathrm{~mm}, I, 0 \mathrm{~mm}, 0,50 \mathrm{~mm}, 0,250 \mathrm{~mm}$ e $0,150 \mathrm{~mm}$. Os teores de $\mathrm{Fe}, \mathrm{SiO}_{2}, \mathrm{Al}_{2} \mathrm{O}_{3}, \mathrm{P}, \mathrm{Mn}$ foram determinados nas faixas supracitadas por fluorescência de raios $X(F R X)$ em um espectrômetro da marca Rigaku, modelo Simultix 14 (Laboratório Químico de Brucutu). A química global foi calculada a partir dos teores analisados em cada faixa. Para realização da análise química as amostras foram dispostas em pastilhas fundidas calcinadas confeccionadas a partir da mistura de uma porção de Ig de minério pulverizado com $5 \mathrm{~g}$ de tetraborato e metaborato de lítio $\left(67 \% \mathrm{Li}_{2} \mathrm{~B}_{4} \mathrm{O}_{7}+33 \% \mathrm{LiBO}_{2}\right)$, levado a fusão a $1.000^{\circ} \mathrm{C}$. A determinação do $\mathrm{PPC}$ foi realizada por gravimetria em forno mufla à temperatura de $1.000^{\circ} \mathrm{C}$ por I hora, pesando-se a amostra antes e depois do aquecimento. A relação da perda de massa com a massa inicial correspondeu ao percentual de PPC.

A análise estatística foi realizada através do software estatístico R [9]. Uma análise descritiva foi realizada buscando conhecer os perfis das variáveis estudadas e posteriormente, foi aplicado o método de análise de agrupamento. $\mathrm{Na}$ execução do script foram utilizados os pacotes cluster [10], factoextra [I I], $\operatorname{devEMF}[12]$ e os pacotes básicos do software $R$.

A avaliação da formação de possíveis grupos com os dados presentes foi feita pela análise hierárquica de agrupamento, na qual se adotou como medida de dissimilaridade a distância euclidiana e como algoritmo de agrupamento o método de Ward $[13,14]$. A partir do número de grupos adotados, a estratificação das amostras foi realizada utilizando-se análise não hierárquica de agrupamento, conhecida como K-means [15].

As características intrínsecas de cada grupo identificado no método K-means foram avaliadas no software R. A disposição geográfica das amostras e a relação destas características com o contexto geológico da jazida, foram avaliados no software de modelagem geológica Vulcan versão I0.I. 


\section{RESULTADOS E DISCUSSÃO}

Algumas análises estatísticas básicas relativas às dez variáveis estudadas são apresentadas na Tabela I. Ao avaliar os coeficientes de variação, que representam o desvio padrão relativo em termos percentuais, nota-se que estes foram significativamente maiores para a faixa granulométrica $+8,0 \mathrm{~mm}$ e para o teor de $\mathrm{Mn}$, indicando grande variabilidade nessas variáveis.

A Figura la apresenta o gráfico boxplot dos dados originais. Porém para os teores de $\mathrm{Al}_{2} \mathrm{O}_{3}, \mathrm{P}, \mathrm{Mn}$ e PPC, por efeito de escala, não foi possível classificar sua variância e visualizar a ocorrência de outliers. Sendo assim, foi realizada a normalização dos dados e os mesmos são apresentados na Figura Ib. Esta normalização tem por finalidade expressar cada observação dos dados originais, em termos de variações inerentes ao sistema, efetuando-se uma transformação dos dados de modo que cada variável apresente média 0 e variância igual a I [16]. Nesta figura observa-se que os dados no geral apresentam grande variabilidade e pode-se detectar a elevada presença de outliers nas faixas granulométricas $+8,0 \mathrm{~mm},-8,0+\mathrm{I}, 0 \mathrm{~mm},-\mathrm{I}, 0+0,15 \mathrm{~mm}$ e nos teores $\mathrm{P}_{,} \mathrm{Al}_{2} \mathrm{O}_{3}$ e principalmente para Mn. Estes outliers foram avaliados, tendo-se constatado que os mesmos são ocorrências reais no depósito de Brucutu e por isso foram considerados na análise de agrupamento posterior.

A primeira etapa de aplicação do método de análise de agrupamento é o cálculo da distância entre os atributos ou variáveis estudados. A escolha do método da medida de dissimilaridade deve levar em consideração o grau de correlação entre as variáveis. Em formações ferríferas é comum os teores de $\mathrm{Fe} \mathrm{e} \mathrm{SiO}_{2}$ apresentarem forte correlação negativa $[17,18]$. Para este trabalho foi identificado que estes componentes químicos apresentaram correlação de $-0,9886$. Assim, foi utilizada a distância euclidiana, como método de dissimilaridade, o qual, conforme Linden [15], não leva em consideração a correlação entre o conjunto de dados.

Para a definição do número ótimo de grupos foi utilizado o gráfico de número de grupos versus o nível de distância do agrupamento de cada estágio do processo (Figura 2), aplicando o algoritmo hierárquico de Ward. A análise do gráfico indicou que a partir de 4 grupos não existem diferenças significativas nos níveis de distância, sendo esta a melhor estratificação dos itabiritos silicosos friáveis. Definido o número de grupos, o método $K$-means foi utilizado na estratificação dos indivíduos para cada agrupamento.

Os grupos formados foram nomeados como Tipologia I (TI), Tipologia 2 (T2), Tipologia 3 (T3) e Tipologia 4 (T4). As principais características de cada grupo, contemplando mínimo, máximo, mediana, média e variância, são mostradas na Tabela 2.

A Tipologia I $(n=1.012)$ apresenta teor de ferro variando de $43,32 \%$ a $61,99 \%$ com média de $54,87 \%$, sendo que esta medida é a maior dentre todos os grupos. Ainda nesta tipologia a média para a granulometria mais fina $(-0,15 \mathrm{~mm})$ foi maior em relação às Tipologias 2 e 4 , sendo também a sua principal fração constituinte. A média do PPC foi ligeiramente superior às demais. Assim, a Tipologia I foi definida como itabirito silicoso friável rico e fino.

Nota-se que as médias dos teores de Fe para as Tipologias 2, 3 e 4 são similares e menores do que a Tipologia I, sendo, então, todas definidas como pobres. A granulometria é a principal característica diferenciadora entre elas.
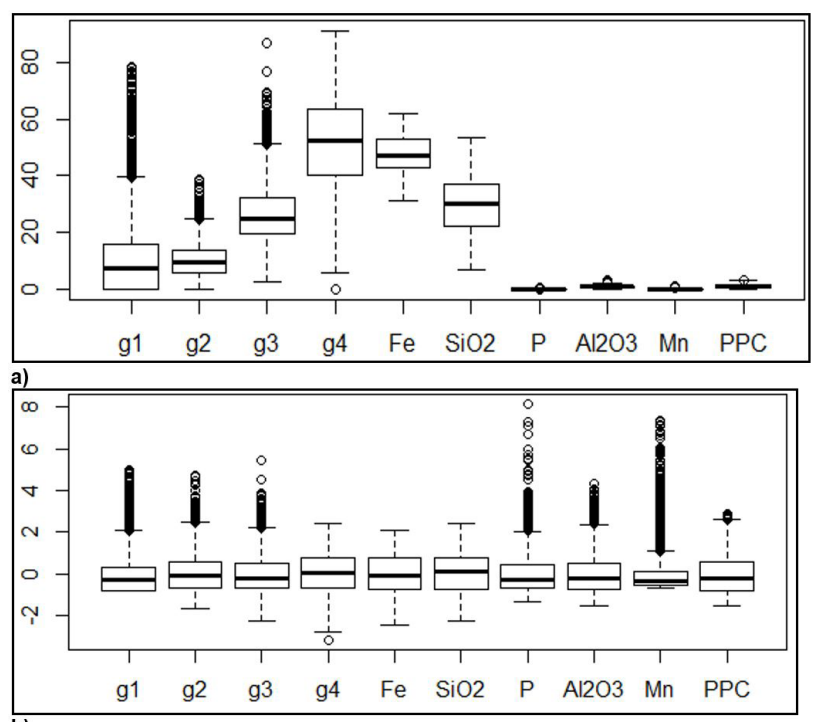

Figura I. Gráfico Boxplot: (a) variáveis da matriz de dados originais; (b) variáveis da matriz de dados normalizados. Sendo: $\mathrm{gl}:+8,0 \mathrm{~mm}$, g2: $-8,0+1,0 \mathrm{~mm}, \mathrm{~g} 3:-\mathrm{I}, 0+0,15 \mathrm{~mm}, \mathrm{~g} 4:-0,15 \mathrm{~mm}$.

Tabela I. Estatística descritiva dos dados originais

\begin{tabular}{|c|c|c|c|c|c|c|}
\hline Variáveis (\%) & Mínimo & Máximo & Mediana & Média & Desvio Padrão & $\begin{array}{c}\text { Coeficiente de } \\
\text { Variação \% }\end{array}$ \\
\hline$+8,0 \mathrm{~mm}$ & 0,00 & 78,54 & 8,88 & 11,37 & 13,46 & 118 \\
\hline$-8,0+\mathrm{I}, 0 \mathrm{~mm}$ & 0,00 & 38,30 & 9,84 & 9,97 & 6,00 & 60 \\
\hline$-1,0+0,15 \mathrm{~mm}$ & 2,40 & 86,96 & 26,34 & 27,22 & 10,90 & 40 \\
\hline$-0,15 \mathrm{~mm}$ & 0,00 & 90,96 & 54,94 & 51,44 & 16,22 & 32 \\
\hline $\mathrm{Fe}$ & 30,97 & 61,99 & 47,90 & 47,78 & 6,83 & 14 \\
\hline $\mathrm{SiO}_{2}$ & 6,86 & 53,58 & 29,32 & 29,51 & 9,92 & 34 \\
\hline$P$ & 0,001 & 0,194 & 0,028 & 0,029 & 0,020 & 70 \\
\hline $\mathrm{Al}_{2} \mathrm{O}_{3}$ & 0,01 & 2,98 & 0,81 & 0,80 & 0,50 & 63 \\
\hline$M n$ & 0,001 & 0,965 & 0,076 & $0,08 \mathrm{I}$ & 0,120 & 148 \\
\hline PPC & 0,09 & 2,99 & 1,12 & $1,1 \mathrm{I}$ & 0,65 & 59 \\
\hline
\end{tabular}


Tabela 2. Estatística descritiva dos grupos formados

\begin{tabular}{|c|c|c|c|c|c|c|c|c|c|c|c|}
\hline Tipologia & Op. & $\begin{array}{c}+8,0 \mathrm{~mm} \\
(\%)\end{array}$ & $\begin{array}{c}-8,0+\mathrm{I}, 0 \mathrm{~mm} \\
(\%)\end{array}$ & $\begin{array}{c}-1,0+0,15 \mathrm{~mm} \\
(\%)\end{array}$ & $\begin{array}{c}-0,15 \mathrm{~mm} \\
(\%)\end{array}$ & $\begin{array}{l}\mathrm{Fe} \\
(\%)\end{array}$ & $\begin{array}{l}\mathrm{SiO}_{2} \\
(\%)\end{array}$ & $\begin{array}{c}P \\
(\%)\end{array}$ & $\begin{array}{c}\mathrm{Al}_{2} \mathrm{O}_{3} \\
(\%)\end{array}$ & $\begin{array}{l}\mathrm{Mn} \\
(\%)\end{array}$ & $\begin{array}{l}\text { PPC } \\
(\%)\end{array}$ \\
\hline TI & $\min$ & 0,00 & 0,00 & 8,45 & 28,05 & 43,32 & 6,86 & 0,001 & 0,01 & 0,002 & 0,19 \\
\hline \multirow[t]{4}{*}{$\mathrm{n}: 1.012$} & $\max$ & 31,58 & 36,92 & 46,60 & 84,72 & 61,99 & 37,41 & 0,173 & 2,98 & 0,862 & 2,99 \\
\hline & med & 8,65 & 12,75 & 22,92 & 55,67 & 54,73 & 19,34 & 0,031 & 0,91 & 0,068 & 1,26 \\
\hline & $\bar{x}$ & 8,85 & 12,99 & 23,03 & 55,12 & 54,87 & 19,14 & 0,030 & 0,94 & 0,072 & $\mathrm{I}, 24$ \\
\hline & var & 42,41 & 31,21 & 33,01 & 91,67 & 17,35 & 36,16 & 0,000 & 0,31 & 0,012 & 0,38 \\
\hline T2 & $\min$ & 0,00 & 0,00 & 21,68 & 0,00 & 31,93 & 11,25 & 0,002 & 0,01 & 0,001 & 0,16 \\
\hline \multirow[t]{4}{*}{$\mathrm{n}: 823$} & $\max$ & 27,25 & 38,30 & 86,96 & 57,44 & 60,27 & 52,39 & 0,173 & 2,97 & 0,956 & 2,97 \\
\hline & med & 7,36 & 9,87 & 41,97 & 40,81 & 44,43 & 34,06 & 0,032 & 0,81 & 0,090 & 1,27 \\
\hline & $\bar{x}$ & 8,03 & $|0,5|$ & 42,09 & 39,37 & 44,97 & 33,36 & 0,032 & 0,83 & 0,086 & $|, 2|$ \\
\hline & var & 47,23 & 41,62 & 90,95 & 92,12 & 26,95 & 54,92 & 0,000 & 0,21 & 0,014 & 0,50 \\
\hline T3 & $\min$ & 0,00 & 0,00 & 9,04 & 47,12 & 31,39 & 10,33 & 0,001 & 0,02 & 0,001 & 0,09 \\
\hline \multirow[t]{4}{*}{$\mathrm{n}: 1.156$} & $\max$ & 26,74 & 20,05 & 41,97 & 90,96 & 61,72 & 52,20 & 0,121 & 2,81 & 0,940 & 2,99 \\
\hline & med & 3,11 & 6,58 & 22,85 & 67,46 & 45,52 & 33,01 & 0,021 & 0,77 & 0,07 I & 0,90 \\
\hline & $\bar{x}$ & 4,15 & 6,48 & 23,12 & 66,25 & 45,08 & 33,64 & 0,021 & 0,76 & 0,082 & 0,88 \\
\hline & var & 31,48 & 17,07 & 34,31 & 77,60 & 25,16 & 51,45 & 0,000 & 0,24 & 0,017 & 0,31 \\
\hline T4 & $\min$ & $|8,7|$ & 0,00 & 2,40 & 5,70 & 30,97 & 8,89 & 0,003 & 0,01 & 0,002 & 0,14 \\
\hline \multirow[t]{4}{*}{$\mathrm{n}: 528$} & $\max$ & 78,54 & 29,86 & 47,75 & 54,64 & 61,64 & 53,58 & 0,194 & 2,98 & 0,965 & 2,98 \\
\hline & med & 35,11 & 10,78 & 20,90 & 33,21 & 44,27 & 34,65 & 0,034 & 0,54 & 0,074 & 1,22 \\
\hline & $\bar{x}$ & 37,22 & $|I, 0|$ & 21,03 & 30,74 & 44,50 & 34,33 & 0,035 & 0,56 & 0,090 & 1,18 \\
\hline & var & 171,54 & 32,37 & 58,29 & 103,23 & 45,85 & 98,97 & 0,000 & 0,14 & 0,015 & 0,50 \\
\hline
\end{tabular}

Nota: n: número de indivíduos, Op: função operacional mínimo (min), máximo (max), mediana (med), média $(\bar{x})$, variância (var).

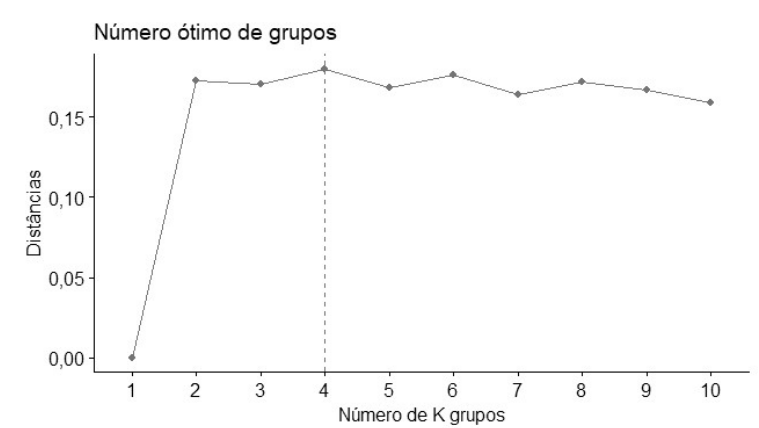

Figura 2. Gráfico com o número ótimo de grupos.

A média e variância da granulometria $-\mathrm{I}, 0+0,15 \mathrm{~mm}$ na Tipologia $2(n=823)$ são consideravelmente as mais elevadas, $42,09 \%$ e $90,95 \%$, respectivamente. Esta foi denominada itabirito silicoso friável pobre e grosseiro.

$\mathrm{Na}$ Tipologia 3 ( $n=1.156)$ destacam-se a média elevada da granulometria $-0,15 \mathrm{~mm}$ de $66,25 \%$, alcançando máximo de $90,96 \%$, e a menor média de PPC dentre os grupos, $0,88 \%$. Em função da comparação de sua granulometria, foi definida como itabirito silicoso friável pobre e fino.

A Tipologia $4(n=528)$ apresenta média significativamente alta para a variável $+8,0 \mathrm{~mm}$ quando comparada aos demais grupos (37,22\%). Amorim e Alkmin [3] descrevem itabiritos semi-compactos como aqueles que apresentam percentual retido em $6,3 \mathrm{~mm}$ entre $30 \%$ a $55 \%$. A partir desta consideração, esta tipologia foi denominada itabirito semi-compacto pobre. A variância para a Tipologia 4 é superior para os atributos $+8,0 \mathrm{~mm},-0,15 \mathrm{~mm}$, Fe e $\mathrm{SiO}_{2}$ quando comparada aos outros grupos, inferindo uma maior heterogeneidade dos dados, que pode estar relacionada com a dificuldade em garantir uma amostragem representativa para este tipo de material, devido a presença de partículas muito grossas, principalmente na amostragem de canaletas em frentes de lavra. Observa-se também que este grupo reúne uma menor quantidade de indivíduos.

As principais variáveis que permitiram a diferenciação dentre os grupos foram então o teor de Fe e os parâmetros granulométricos, sendo estas informações sintetizadas no gráfico da Figura 3.

Analisando estas tipologias no depósito de Brucutu, observa-se uma íntima relação das mesmas para com a geologia da jazida, conforme ilustrado na Figura 4.

A Tipologia I (Figura 4a) com teor de Fe e PPC mais elevados ( $\mathrm{Fe}=54,87 \%$ e PPC $=1,24 \%$ ), apresenta-se com maior concentração de indivíduos na região próxima à superfície natural do depósito. Nesta região é esperado que as formações ferríferas apresentem teores de Fe e PPC mais altos, uma vez que estas perfazem o horizonte de maior ação do intemperismo, o qual proporciona o enriquecimento supergênico [6]. A Tipologia 2 (Figura 4b), em termos geológicos, é fortemente marcada pelo agrupamento dos indivíduos na direção NW-SE, na parte central e oeste da mina. Estas duas faixas de concentração para esta tipologia são coincidentes com as direções axiais NW-SE das dobras, propostas por Endo et al. [8], como registro da fase de dobramento progressivo do evento brasiliano presente no depósito. 


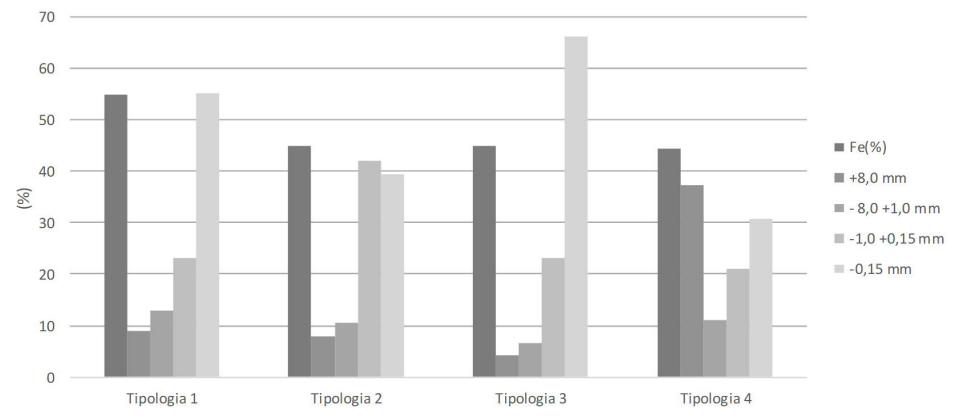

Figura 3. Gráfico com os agrupamentos formados e suas principais variáveis diferenciadoras.
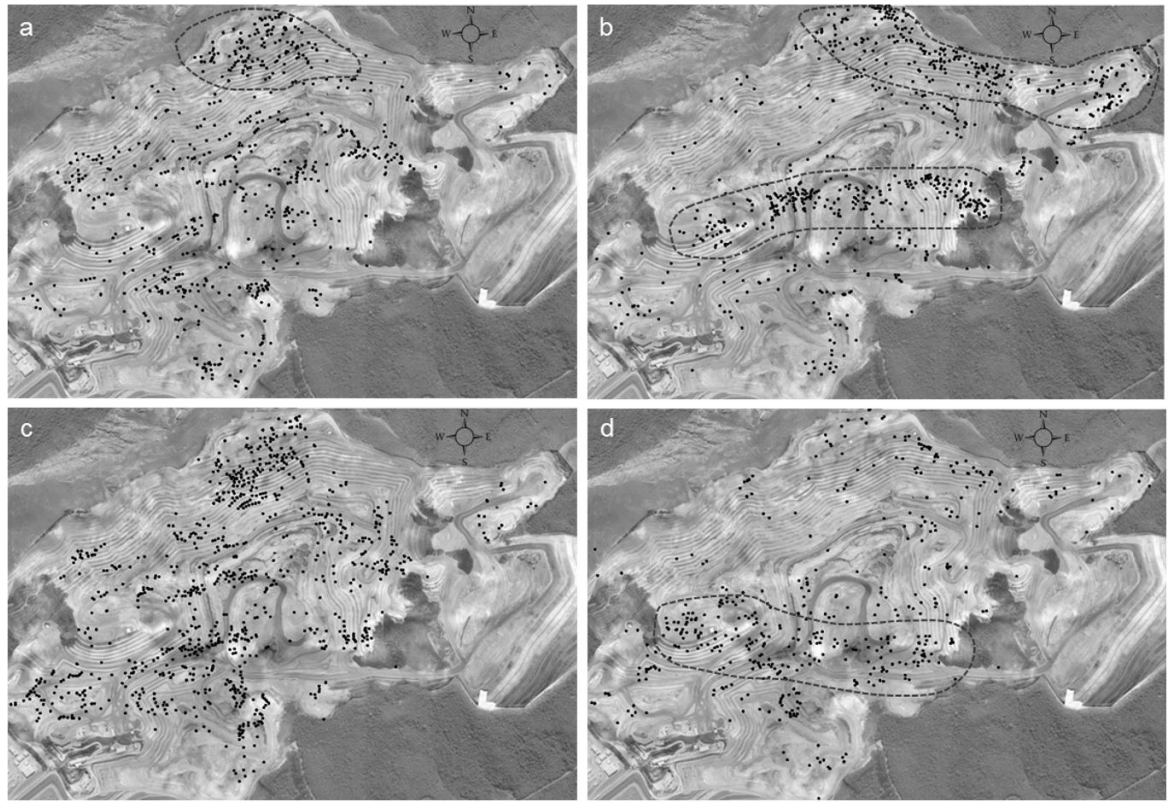

Figura 4. Distribuição espacial das diferentes tipologias de itabiritos silicosos friáveis no depósito de Brucutu. (a) Tipologia I, (b) Tipologia 2, (c) Tipologia 3 e (d) Tipologia 4.

A distribuição espacial da Tipologia 3 (Figura 4c) é dispersa ao longo do depósito sendo que, a priori, não se identifica uma relação geológica clara para com a mesma. Para a tipologia 4 (Figura 4d), aqui definida como semi-compacta, os indivíduos apresentam-se agrupados na porção mais sudoeste da mina onde é perceptível in loco a presença de materiais mais granulados e semi-compactos os quais são característicos das regiões intituladas na mina como Gralhos Oeste e Vargem da Lua.

\section{CONCLUSÃO}

Em função dos teores de $\mathrm{Fe}, \mathrm{SiO}_{2}, \mathrm{Al}_{2} \mathrm{O}_{3}, \mathrm{P}, \mathrm{Mn}$ e PPC e distribuição granulométrica das amostras, os minérios Itabiritos Silicosos Friáveis da Mina de Brucutu podem ser subdivididos em quatro tipologias homogêneas classificadas e descritas como:

I) Tipologia I - itabirito silicoso friável rico e fino: representa os itabiritos ricos, sendo estes produtos do enriquecimento supergênico, dado sua localização geográfica no topo do depósito e estratigráfica acima das rochas intrusivas máficas;

2) Tipologia 2 - itabirito silicoso friável pobre e grosseiro: agrupamento marcado pela direção NW-SE a qual coincide com a direção axial da fase do dobramento brasiliano;

3) Tipologia 3 - itabirito silicoso friável pobre e fino: dispersa ao longo do depósito, dificultando correlacionar a mesma com alguma fase geológica;

4) Tipologia 4 - itabirito semi-compacto pobre: demarcada pela concentração na região SW do depósito, onde, in loco, é característica a presença de itabiritos compactos.

\section{Agradecimentos}

Os autores agradecem ao Programa de Pós-Graduação em Engenharia Mineral/UFOP, CAPES, CNPq, Fapemig e VALE. 


\section{REFERÊNCIAS}

I Luz AB, Lins FF. Introdução ao tratamento de minérios. In: Luz AB, Sampaio JA, França, SCA. Tratamento de minérios. 5. ed. Rio de Janeiro: CETEM/MCP; 20I0. p. 3-I5.

2 Alkmim AR. Investigação geoquímica e estratigráfica da formação ferrífera Cauê na porção centro-oriental do Quadrilátero Ferrífero [dissertação]. Ouro Preto: Universidade Federal de Ouro; 2014.

3 Amorim LQ, Alkmin FF. New ore types from Cauê Banded Iron Formation Quadrilátero Ferrífero, Minas Gerais, Brazil - Responses to the growing demand. In: Proceedings of the Iron Ores Conference; 20I I; Perth, WA. Nova Zelândia: The Australasian Institute of Mining and Metallurgy; 20I I. p. 59-7I.

4 Roberto JB. Influência dos diversos tipos litológicos nas operações de concentração da instalação de beneficiamento de Brucutu [dissertação]. Belo Horizonte: Universidade Federal de Minas Gerais; 2010.

5 Rocha JMP. Definição da tipologia e caracterização mineralógica e microestrutural dos itabiritos anfibolíticos das Minas de Alegria da Samarco Mineração S.A [tese]. Belo Horizonte: Universidade Federal de Minas Gerais; 2008.

6 Sousa DVM. Estudo geoquímico-mineral das formações ferríferas bandadas do Sinclinal Gandarela, Quadrilátero Ferrífero (MG) [dissertação]. Ouro Preto: Universidade Federal de Ouro Preto; 2016.

7 Hagemann SG, Angerer T, Duuring P, Rosière CA, Figueiredo e Silva RC, Lobato L, et al. BIF - hosted iron mineral system: a review. Ore Geology Reviews. 2016;76:317-359.

8 Endo I, Silva AG, Mariano DF, Espinoza JAA, Lopes AP, Angeli G. Estratigrafia e arcabouço estrutural dos distritos ferríferos de Brucutu e Dois Irmãos, Quadrilátero Ferrífero, MG. Ouro Preto: Convênio Vale - Universidade Federal de Ouro Preto e Fundação Gorceix; 2008. Relatório Final Vale (Projeto Brucutu-Dois Irmãos).

9 R Core Team. R: a language and environment for statistical computing. Vienna: R Foundation for Statistical Computing; 2017 [acesso em 19 set. 2018]. Disponível em: https://www.R- project.org/

10 Maechler M, Rousseeuw P, Struyf A, Hubert M, Hornik K. Cluster: cluster analysis basics and extensions. R package version 2.0.6. Vienna: R Development Core Team; 2017.

II Kassambara A, Mundt F. Factoextra: extract and visualize the results of multivariate data analyses. R package version I.0.5. Vienna: R Development Core Team; 2017 [acesso em 19 set. 2018]. Disponível em: https://CRAN.R-project. org/package $=$ factoextra

12 Johnson P. devEMF: EMF Graphics Output Device. R package. Version 3.6-2. Vienna: R Development Core Team; 2019 [acesso em 19 set. 2018]. Disponível em: https://CRAN.R-project.org/package = devEMF

I3 Pereira TM. Estatística multivariada I - Uma abordagem aplicada utilizando o software R. Ouro Preto: Departamento de Estatística, Universidade Federal de Ouro Preto; 2017. Notas de aula.

I 4 Lindsey CR, Neupane G, Spycher N, Fairley JP, Dobson P, Wood T, et al. Cluster analysis as a tool for evaluating the exploration potential of Known Geothermal Resource Areas. Geothermics. 2018;72:358-370.

15 Linden R. Técnicas de agrupamento. Revista de Sistemas de Informações da FSMA. 2009;4: 18-36.

16 Moita JM No, Moita GC. Uma introdução à análise exploratória de dados multivariado. Química Nova. 1998;2I (4):467-469.

17 Raposo FO. Formações ferríferas e metassedimentos químicos de áreas selecionadas do quadrilátero ferrífero e suas relações com níveis rudáceos [dissertação]. Belo Horizonte: Universidade Federal de Minas Gerais; 1996.

18 Girelli E, Bassani M, Chatelier NL, Costa RA, Vicenzi R. Estudo de previabilidade de um depósito de ferro [trabalho de conclusão de curso]. Porto Alegre: Universidade Federal do Rio Grande do Sul; 2012.

Recebido em: 19 Set. 2018

Aceito em: 4 Maio. 2019 